Reprod. Nutr. Dévelop. 1980, 20 (2), 539-544.

\title{
Quelques aspects du contrôle hormonal de la synthèse de la vitellogénine chez le crustacé amphipode Orchestia gammarella (Pallas)
}

\author{
par Marie-Françoise BLANCHET, Hélène CHARNIAUX-COTTON, Y. CROISILLE *, \\ Henriette JUNERA, Madeleine MARTIN, J.-J. MEUSY, C. ZERBIB \\ Laboratoire de Sexualité et Reproduction des Invertébrés, \\ Université Pierre-et-Marie-Curie, Bât. A, \\ 4, place Jussieu, 75230 Paris Cedex 05. \\ * Institut d'Embryologie du C.N.R.S. et du Collège de France. \\ 49 bis, av. de la Belle Gabrielle, 94130 Nogent-sur-Marne.
}

Summary. Some aspects of hormonal control of vitellogenin synthesis in Orchestia gammarella (Pallos) (Crustacea, Amphipoda).

The presence of a vitellogenin stimulating ovarian hormone (VSOH) in Orchestio gammarella has been demonstrated by gonadectomy and ovary grafting. Vitellogenin synthesis is not affected by molting hormone injection ; it decreases after $Y$-organ (molting gland) cauterization. Studies in progress seem to indicate that the destruction of the median area of the protocerebrum is followed by a decrease of this synthesis.

\section{Introduction}

La vitellogénine est un complexe lipo-glyco-caroténoprotéique de haut poids moléculaire, spécifique du milieu intérieur des femelles en reproduction de Crustacés, Myriapodes, Insectes, Amphibiens, Oiseaux ef de certains Poissons. La vitellogénine est synthétisée hors des ovaires (par le corps gras chez les Insectes, par le foie chez les Vertébrés) et constitue le principal précurseur du vitellus protéique.

Nous limiterons cet exposé au contrôle hormonal de la synthèse de la vitellogénine chez l'Amphipode Orchestia gammarella, renvoyant pour les aspects généraux à la revue de l'un de nous (Meusy, 1980).

\section{Vitellogénine et hormones sexuelles}

1. - Induction de la synthèse de la vitellogénine chez le mâle. - Chez les Oiseaux et les Amphibiens, la synthèse de la vitellogénine est inductible dans le foie du mâle par action de l'œstradiol-17 $\beta$. 
Nous avons cherché à induire in vivo cette synthèse chez les mâles d'O. gammarella. Nous avons d'abord privé ces sujets de leurs glandes androgènes : la vitellogénine n'apparaît jamais dans l'hémolymphe (Meusy, Junéra et Croisille, 1971). Rappelons que l'hormone androgène $a$ un effet inhibiteur à la fois sur la vitellogenèse (CharniauxCotton, 1957) et sur la synthèse de la vitellogénine lorsqu'on la fait intervenir expérimentalement chez des femelles (Meusy, Junéra ef Croisille, 1971) ; le même résultat a été obtenu chez les Isopodes (Besse et al., 1970). Pour obtenir la synthèse de la vitellogénine chez les mâles privés de leurs glandes androgènes, il est nécessaire de leur greffer un ovaire (Meusy, Junéra et Croisille, 1971) (fig. 1). Chez de tels sujets, les testicules ne s'inversent jamais en ovaires fonctionnels : la spermatogenèse s'arrête, mais les ovocytes qui apparaissent exceptionnellement ne se développent pas.

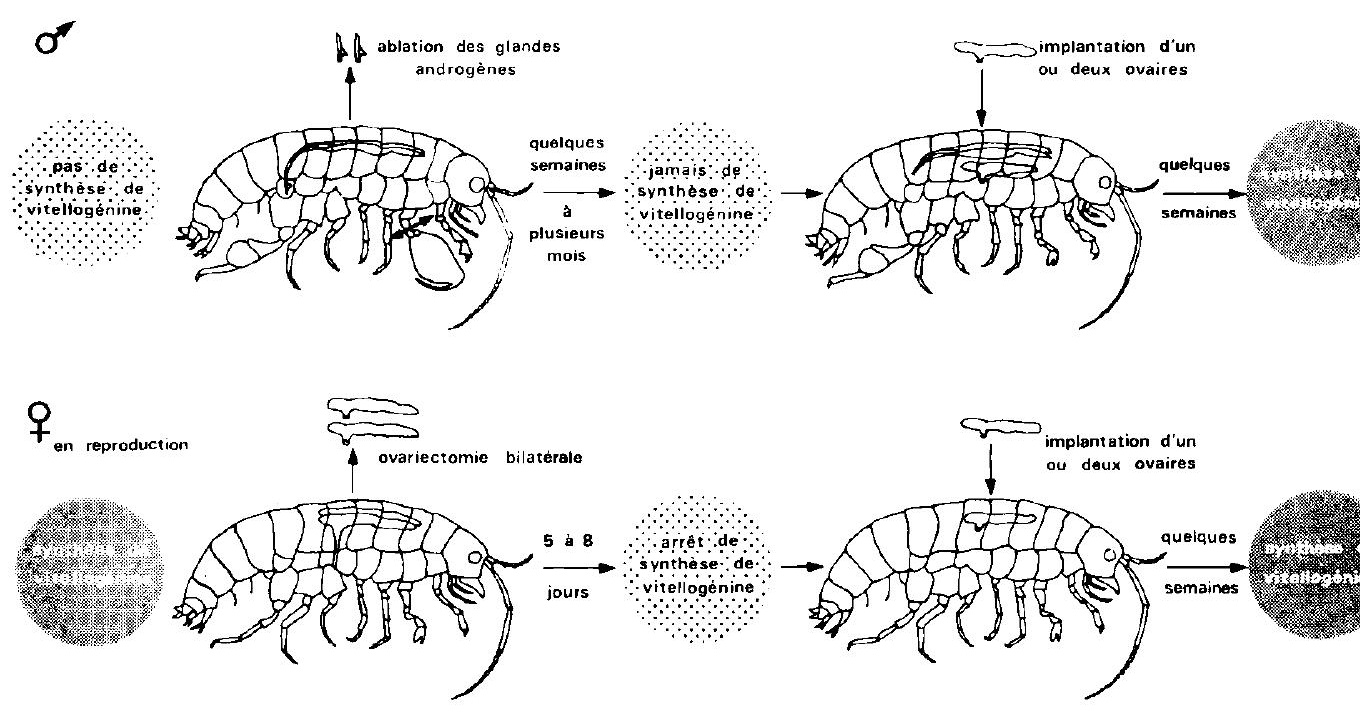

FIG. 1. - Expériences ayant permis de prouver l'exisfence d'un contrôle hormonal par l'ovaire de la synthèse de la vifellogénine chez Orchestia gammarella (d'après Meusy, 1978, modifié). Noter que, chez le mâle privé de ses glandes androgènes, le $2^{\mathrm{e}}$ gnathopode régénère après section sous la forme femelle. Ceci permet de s'assurer qu'aucune fraction de glande androgène n'est demeurée en place.

2. - Contrôle de la synthèse de la vitellogénine chez les femelles. - Après ovariectomie bilatérale des femelles d'O. gammarella en période de reproduction, la synthèse de la vitellogénine s'arrête complètement après un délai de 5 à 8 jours (Junéra et al., 1977) (fig. 2). La greffe d'un ovaire rétablit la synthèse de la vitellogénine chez les femelles ovariectomisées.

Ces résultats prouvent :

- que la vitellogénine, dont le lieu de synthèse n'est pas encore clairement établi chez les Crustacés, est d'origine extra-ovarienne (s'il en était autrement, la synthèse s'arrêterait immédiatement après l'ovariectomie); 
- que l'ovaire produit une hormone qui contrôle la synthèse de la vitellogénine (ou, éventuellement, sa libération). Nous avons nommé cette hormone «VSOH », c'est-àdire «Vitellogenin Stimulating Ovarian Hormone».

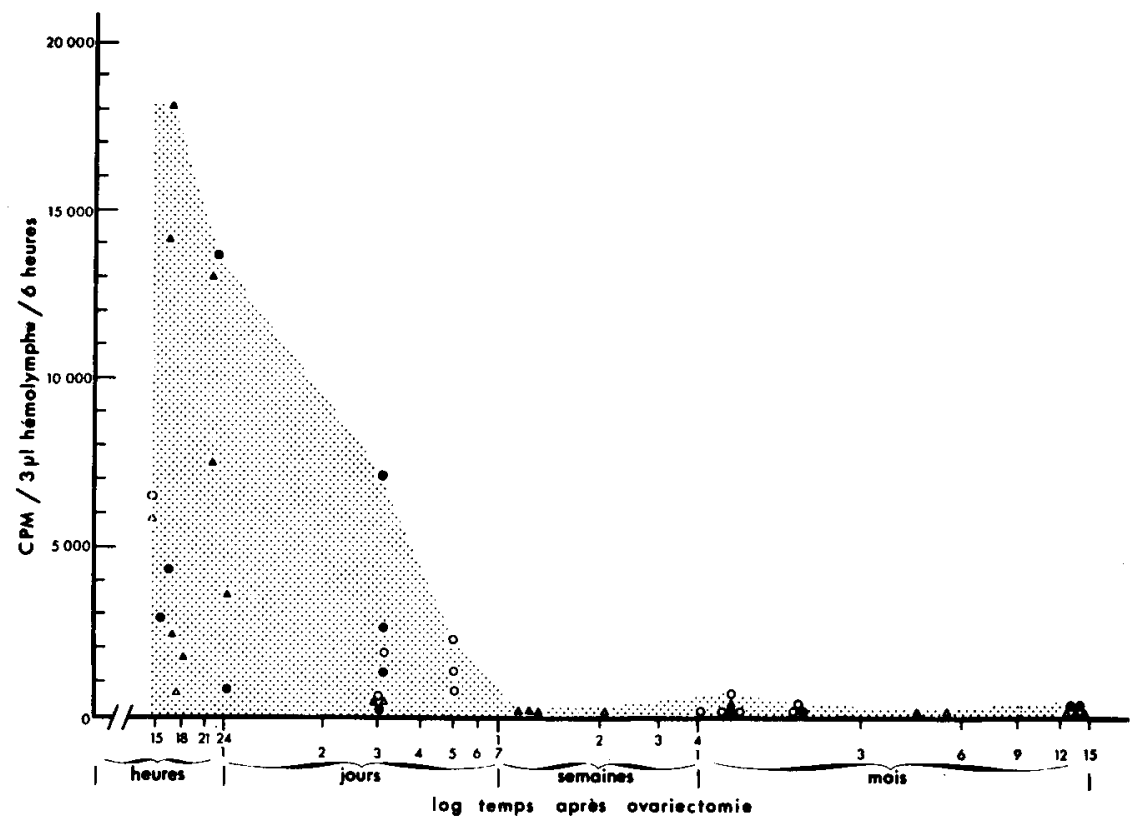

FIG. 2. - Effet de l'ovariectomie bilatérale sur la synthèse de la vitellogénine chez Orchestia gammarella (d'après Junéra et al., 1977). Les animaux étudiés ont reçu une injection de leucine ${ }^{3} \mathrm{H} 6 \mathrm{~h}$ avant la prise d'hémolymphe. Après disc-électrophorèse, la radioactivité a été mesurée au niveau de la vitellogénine et rapportée à $3 \mu \mathrm{l}$ d'hémolymphe. Tous les points correspondent d̀ des animaux distincts, étudiés à différents stades de l'intermue. $(\Delta)$, stade $C ;(\Lambda)$, stade $D_{0} ;(0)$, stade $D_{1}$, ; $(\bullet)$, stade D.

3. - Discussion et conclusions. - L'existence d'une hormone sexuelle contrôlant positivement la synthèse de la vitellogénine n'avait jamais été mise en évidence auparavant chez les Crustacés. Nous ignorons actuellement si la VSOH est présente chez tous les Crustacés Malacostracés. Chez l'Isopode Porcellio dilatatus, l'ovariectomie conduit, d'après Picaud (1974), à une accumulation de la vitellogénine dans l'hémolymphe ; en l'absence de données concernant l'incorporation d'un précurseur radioactif, on ignore si la synthèse de la vifellogénine décroît ou éventuellement s'arrête après un délai plus ou moins long.

Chez les Insectes, où la synthèse de la vitellogénine est généralement induite et régulée par l'hormone juvénile, les ovaires de quelques espèces, notamment la Drosophile (Kambysellis, 1977), semblent sécréter une hormone homologue à la VSOH. Rappelons aussi que l'œstradiol-17 $\beta$, dont le rôle sur la synthèse de la vitellogénine chez les Poissons, les Amphibiens et les Oiseaux est clairement établi, est également une hormone d'origine ovarienne. 


\section{Vitellogénine et hormone de mue}

L'hormone de mue joue-t-elle un rôle dans la synthèse de la vitellogénine qui apparaît comme un processus lié au cycle d'intermue (Meusy, Junéra ef Croisille, 1974)?

Deux types d'expériences ont été réalisés chez 0 . gammarella : implantation de cristaux d'ecdystérone et cautérisation des organes $Y$ (glandes de mue).

1. - Introduction de cristaux d'ecdystérone. - L'ecdystérone (200 ng/mg poids frais) ne déclenche pas la synthèse de la vitellogénine lorsqu'elle est introduite en début d'intermue ; administrée plus tardivement, elle n'affecte pas le taux de cette synthèse (Blanchet, Junéra et Meusy, 1975).

2. - Cautérisation des organes $Y$. - La cautérisation des organes $Y$ provoque une dépression de la synthèse de la vitellogénine. Corrélativement, cette opération entraîne un arrêt de la croissance des ovocytes en viłellogenèse (Meusy, Blanchet ef Junéra, 1977).

3. - Discussion ef conclusions. - A l'exception du cas, semble-t-il particulier, du Moustique Aedes aegypti (Hagedorn et Fallon, 1973 ; Hagedorn et al., 1975), l'hormone de mue ne paraît pas constituer un facteur possédant une action spécifique sur la synthèse de la vitellogénine chez les Arthropodes. Différents auteurs ont d'ailleurs mis en évi-

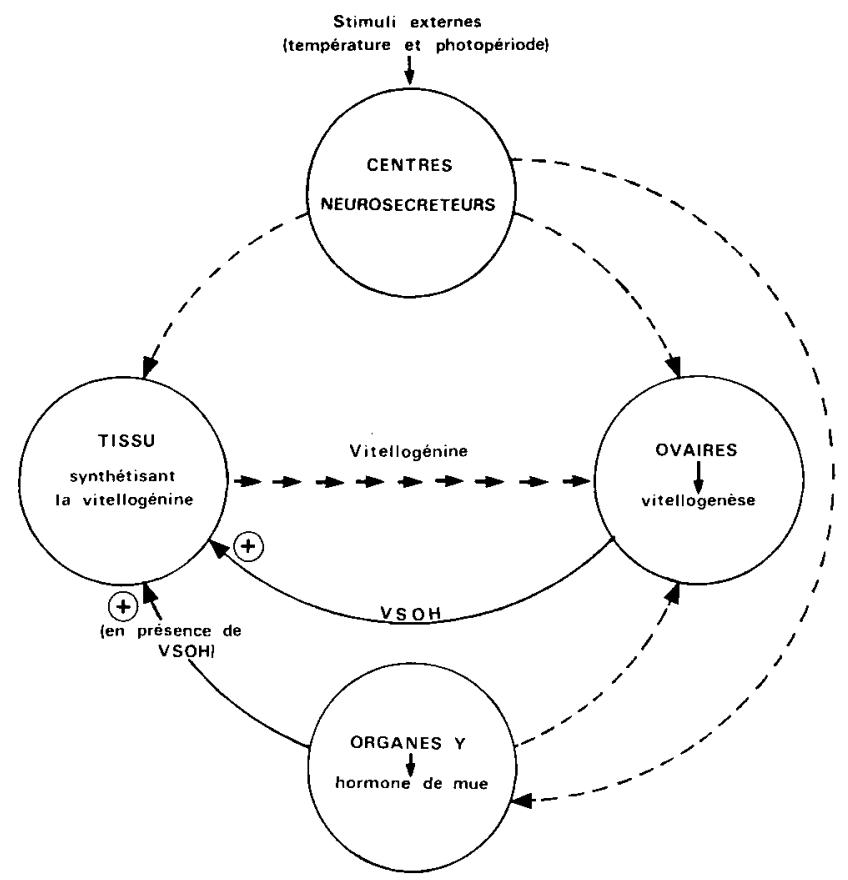

FIG. 3. - Représentation schématique du contrôle endocrine de la synthèse de la vifellogénine chez Orchestia gammarella. $\longrightarrow$, contrôles établis ; $-\cdots \rightarrow$, contrôles hypothétiques. 
dence des effets stimulants de cette hormone sur divers métabolismes, liés ou non à la mue (revue in Slama, 1974). Il semble que la dépression de la synthèse de la vitellogénine observée chez $O$. gammarella, après destruction des organes $Y$, doive également être envisagée dans le cadre des actions non spécifiques de l'ecdystérone.

\section{Vitellogénine et neurosécrétion}

Il est bien établi que la vitellogenèse est contrôlée chez les Crustacés par des mécanismes neurosécrétoires, mais l'action des neurohormones sur la synthèse de la vitellogénine est pratiquement inconnue. Les premiers résultats de travaux en cours dans notre laboratoire paraissent indiquer que la destruction de la région médiane du protocérébron des femelles d'O. gammarella est suivie d'une diminution du taux de la synthèse de la vitellogénine.

Nous chercherons à savoir si les neurohormones cérébrales interviennent directement sur cette synthèse ou agissent en contrôlant la sécrétion de la VSOH par les ovaires (fig. 3).

Présenté ou Colloque D.G.R.S.T. de Port Bail, 27 février-1 er mars 1979.

Accepté en septembre 1979.

Remerciements. - Travail réalisé avec l'aide de la DGRST (contrat no 78.7.2743).

\section{Références}

BESSE G., JUCHAULT P., LEGRAND J.-J., MOCQUARD J.-P., 1970. Modification de l'électrophorégramme de l'hémolymphe des Oniscoïdes (Crustacés Isopodes) par action de l'hormone androgène. C. R. Acad. Sci. Paris, sér. D, 270, 3276-3279.

BLANCHET M.-F., JUNERA H., MEUSY J.-J., 1975. Mue et vitellogenèse chez Orchestia gammarella Pallas (Crustacé Amphipode) : étude de la synthèse de la fraction protéique femelle après introduction d'ecdystérone. Experientia, 31, 865-867.

CHARNIAUX-COTTON H., 1957. Croissance, régénération et déterminisme endocrinien des caractères sexuels d'Orchestia gammarella Pallas (Crustacé Amphipode). Ann. Sci. nat., 19, 411-560.

HAGEDORN H. H., FALLON A. M., 1973. Ovarian control of vitellogenin synthesis by the fat body in Aedes aegypti. Nature, 244, 103-105.

HAGEDORN H. H., O'CONNOR J. D., FUCHS M. S., SAGE B., SCHLAEGER D. A., BOHM M. K., 1975. The ovary as a source of $\alpha$-ecdysone in an adult mosquito. Proc. Nat. Acad. Sci., USA, 72, 3255-3259.

JUNERA H., ZERBIB C., MARTIN M., MEUSY J.-J., 1977. Evidence for control of vitellogenin synthesis by an ovarian hormone in Orchestia gammarella (Pallas), Crustacea ; Amphipoda. Gen. comp. Endocrinol., 31, 457-462.

KAMBYSELLIS M. P., 1977. Genetic and hormonal regulation of vitellogenesis in Drosophila. Amer. Zool., 17, 535-549.

MEUSY J.-J., 1978. La formation du vitellus chez les Crustacés. La Recherche, 9, 388-389.

MEUSY J.-J., 1980. Vitellogenin, the extraovarian precursor of the protein yolk in Crustacea : A review. Reprod. Nutr. Dévelop., 20, 1-21.

MEUSY J.-J., BLANCHET M.-F., JUNERA H., 1977. Mue et vitellogenèse chez le Crustacé Amphipode Orchestia gammarella Pallas. II. Ełude de la synthèse de la vitellogénine (« fraction protéique femelle » de l'hémolymphe) après destruction des organes Y. Gen. comp. Endocrinol., $33,35-40$. 
MEUSY J.-J., JUNÉRA H., CROISILLE Y., 1971. Recherche de la « fraction protéique femelle " chez les Crustacés Amphipodes Orchestia gammarella ayant subi une inversion expérimentale du sexe. C. R. Acad. Sci. Paris, sér. D, 273, 592-594.

MEUSY J.-J., JUNÉRA H., CROISILLE Y., 1974. Données sur la synthèse de la fraction protéique femelle chez Orchestia gammarella Pallas (Crustacé Amphipode), au cours de l'intermue et chez les femelles en repos sexuel. C. R. Acad. Sci. Paris, sér. D, 279, 587-590.

PICAUD J.-L., 1974. Contribution à l'étude par électrophorèse des protéines des Oniscoïdes. Th. 3 e cycle, Poitiers.

SLAMA K., 1974. Physiological effects of ecdysoids. In Slama K., ROMANUK M., SORM F., Insect hormones and bioanalogues. Springer-Verlag, Wien-New York. 